\title{
A HABILIDADE DO GESTOR CFO E SUA RELAÇÃO COM A EFICIÊNCIA DAS EMPRESAS LISTADAS NA BOLSA DE VALORES BRASILEIRA
}

\section{CFO MANAGER'S SKILL AND ITS RELATIONSHIP TO THE EFFICIENCY OF COMPANIES LISTED IN THE BRAZILIAN STOCK EXCHANGE}

0 artigo foi aprovado e apresentado no XIX USP International Conference in Accounting, realizado de 24/07 a 26/07 de 2019, em São Paulo (SP).

\section{RESUMO}

Este estudo analisou o efeito da capacidade gerencial do diretor financeiro (CFO) na qualidade dos resultados divulgados pelas firmas listadas na B3. Para obter a capacidade gerencial do CFO foi utilizada a abordagem de Demerjian et al. (2013), na qual se estima a eficiência total da empresa mediante um método de fronteira eficiente e o resíduo da equação é tomado para criação da medida. Foi utilizada a reapresentação dos resultados contábeis como proxy de qualidade de resultados da firma. Foi considerado como eficiência da firma a capacidade de agregar valor aos insumos adquiridos ou consumidos. A eficiência foi estimada através do método de Stochastic Production Frontier (SFA), mediante um fixed effects production frontier model. Para verificar se a parcela de eficiência da firma atribuível a capacidade do $\mathrm{CFO}$ e a sua equipe tem relação com a qualidade dos resultados foi estimado um modelo de regressão probit em painel mediante o generalized estimating equation (GEE) approach para descobrir o efeito médio na população da variável que representa a capacidade gerencial, Mhabil, em relação ao efeito específico individual. As evidências empíricas indicam que maior capacidade gerencial do CFO tem relação negativa com a probabilidade de reapresentação dos relatórios financeiros e, consequentemente, maior a qualidade dos resultados para as empresas. Os resultados indicam que a escolha do gestor CFO é importante para o mercado de capitais, demonstrando que CFOs mais capazes implicam em menores chances de reapresentação dos resultados financeiros. Para a análise foram utilizadas informações anuais das empresas brasileiras listadas na B3 no período de 2010 a 2017.

Palavras-Chave: Eficiência da firma; Habilidade do gestor; Qualidade de resultados.

\begin{abstract}
This study analyzed the effect of CFO management on the quality of results reported by firms listed in B3. To obtain the CFO managerial capacity, the approach of Demerjian et al. (2013), in which the total efficiency of the company is estimated using an efficient boundary method and the residue of the equation is taken to create the measure. The restatement of accounting results was used as a proxy for the firm's earnings quality. The efficiency of the firm is considered as the ability to add value to inputs purchased or consumed. Efficiency was estimated using the Stochastic Production Frontier (SFA) method using a fixed effects production frontier model. To verify whether the firm's efficiency share attributable to the CFO's capacity and its team is related to the quality of results, a panel probit regression model was estimated using the generalized estimating equation (GEE) approach to find out the average effect on the population of the variable that represents managerial capacity, Mhabil, in relation to the individual specific effect. Empirical evidence indicates that greater CFO managerial capacity has a negative relationship with the likelihood of restatement of financial reports and, consequently, higher quality of results for companies. The results indicate that the choice of CFO manager is important for the capital market, demonstrating that more capable CFOs imply lower chances of restating financial results. For the analysis we used annual information of the Brazilian companies listed in B3 from 2010 to 2017.
\end{abstract}

Keywords: Firm efficiency; Manager's ability; Quality of results.
Jose Antonio Tejeda Almonte

Visiting Reseacher at Zicklin Business School at Baruch College. Doutorando em Ciências Contábeis e Administração pela FUCAPE Business School. Mestre em Ciências Contábeis pela Universidade Federal da Bahia (UFBA). Graduado em Contabilidad mención Finanzas pela Universidad Acción pro Educación y Cultura (UNAPEC). Contato: Glenn Road 25 West Orange, NJ, CEP: 07052. E-mail: jtejedaalmonte@gmail.com.

\section{Andréia Hartwig \\ Doutoranda em Contabilidade e Administração e Bacharel em Ciências Contábeis pela Fucape Business School Contato: Av. Fernando Ferrari, 1358. Boa Vista, Vitória/ES. CEP 29.075-505. E-mail: andreiahartwig@aluno.fucape.br.}

\section{Taianan Alves Uzeda Luna} Mestra em Ciências Contábeis pela Universidade Federal da Bahia (UFBA) Graduada em Ciências Contábeis pela Universidade Federal da Bahia (UFBA) Professora da Faculdade Adventista da Bahia. Contato: Rua Carlos Mariguella, 836 d, S. Marcos, CEP: 41.250-570, Salvador/BA. E-mail: taianan.uzeda@hotmail.com. 


\section{INTRODUÇÃO}

O capital humano é uma das principais fontes de geração de valor dentro do ambiente organizacional. Neste sentido, a habilidade gerencial é um dos fatores mais importante na firma (Gong, Law, Chang \& Xin, 2009). Gerentes mais capazes são aqueles que possuem melhor conhecimento operacional, fazendo melhores julgamentos e tomando decisões de maneira mais eficiente (Demerjian, Lev \& Mcvay, 2012). Este trabalho tem como objetivo analisar o efeito da capacidade gerencial do diretor financeiro, aqui denominado $\mathrm{CFO}$, na qualidade dos resultados financeiros das firmas listadas na bolsa de valores brasileira B3 Brasil, Bolsa e Balcão. Desta forma, pretende-se verificar em empresas de diversos setores se maior capacidade gerencial do CFO remete a uma melhor qualidade dos resultados contábeis.

Com base em pesquisa passada, espera-se encontrar uma relação positiva entre a capacidade gerencial do CFO e qualidade dos resultados financeiros divulgados pelas empresas (Demerjian, Lev, Lewis \& Mcvay, 2013). De maneira específica, espera-se que os gestores com maior capacidade gerencial obtenham menor volume de reapresentações das demonstrações financeiras. Nesta pesquisa será utilizada reapresentações dos relatórios contábeis como proxy de baixa qualidade de resultados, pois as pesquisas apontam que empresas que possuem equipes gerencias mais hábeis divulgam com maior acurácia a realidade operacional da firma.

Gestores mais capazes são mais hábeis em elaborar estimativas confiáveis sobre o futuro da empresa, reportando com isso melhores resultados (Demerjian et al., 2012; Demerjian et al. 2013). Tomando como medida de performance a qualidade dos resultados financeiros divulgados pela firma e controlando o efeito relacionado as características de tamanho da companhia, disponibilidade de caixa e alavancagem, os gerentes capazes de estimar de maneira mais acurada provisões, taxas de amortizações e depreciações e valor justo dos ativos obtém melhores resultados financeiros. Embora exista uma vasta literatura da área de qualidade dos resultados, estes se concentram principalmente nas características específicas da empresa, tais como o efeito do comitê de auditoria (Becker, 1998), board interlocking (Muzatko \& Teclezion, 2016) e stakeholders (Miragaia, Ferreira \& Ratten, 2017).

Este estudo examina o aspecto específico do gerente na qualidade dos resultados. É importante ressaltar que apesar de existir alguns trabalhos sobre o efeito da capacidade da gerência financeira na qualidade dos resultados na literatura internacional, as pesquisas no Brasil se concentram nos aspectos do diretor executivo, denominado aqui como CEO, tais como: reputação do $\mathrm{CEO}$, troca do $\mathrm{CEO}$, relação do CEO no desempenho e características do CEO no gerenciamento de resultados (Maestri \& Malaquias, 2018; Sprenger, Krombauer \& Costa, 2017; Matos, Penna \& Da Silva, 2015). Estes trabalhos não abordam como a habilidade do CFO afeta o processo de preparação dos relatórios contábeis.

Este estudo segue a linha de Ge, Matsumoto e Zhang (2008), que encontraram evidências que CFOs mais velhos são mais conservadores nas estimativas divulgadas em demonstrações financeiras. Ge, Matsumoto e Zhang (2011) detectaram a influência dos estilos individuais dos gestores nas escolhas contábeis mediante duas dimensões: ferramentas contábeis que os CFOs podem escolher para atingir as metas dos relatórios financeiros e medidas baseadas em resultados para capturar as propriedades dos ganhos. Também, segue a linha de Xu e Zhao (2016) que identificaram que empresas em período de reapresentação dos relatórios contábeis tendem a contratar CFOs com maior conhecimento contábil e experiência de trabalho.

A percepção geral do trabalho é que existe uma relação positiva entre a capacidade gerencial do CFO e a qualidade dos resultados (Demerjian et al. 2013), mas no Brasil o efeito difere em magnitude pela conjunção de duas razões. Primeiro, a literatura internacional aponta que, na ocorrência de irregularidades contábeis, CFOs são mais propensos a assumir a culpa quando o CEO é um dos fundadores (Leone \& Liu, 2010). Segundo, a maioria das empresas listadas na bolsa de valores brasileira é controlada pelos fundadores ou seus familiares (Burkart, Panunzi \& Shleifer, 2003). Veja que é de sumo interesse o estudo das relações entre a qualidade dos resultados e a capacidade do CFO no caso da existência de maior influência dos CEOs sobre os CFOs, arrojando novos insights sobre a capacidade dos últimos na elaboração dos relatórios financeiros dentro do contexto brasileiro.

Foi feita uma busca nas revistas acadêmicas no Brasil e foi constatado que existe uma lacuna de pesquisa sobre a habilidade do CFO e sua relação no processo de preparação dos relatórios financeiros no mercado de capitais brasileiro. Dado que este é um tema inexistente e levando em consideração as características de controle presentes no âmbito brasileiro, o presente estudo busca preencher essa lacuna identificada. A relação positiva esperada entre qualidade dos resultados e capacidade do CFO é baseada na hipótese de que os CFOs têm uma quantidade substancial de controle sobre os resultados reportados pela empresa (Geiger \& North, 2006). O trabalho se concentra no CFO, porque este supervisiona o processo de criação das demonstrações financeiras e possui maior poder de influir diretamente nas decisões contábeis relacionadas a empresa.

Para o alcance do objetivo da pesquisa, foi estimada por meio de regressão a capacidade gerencial do CFO seguindo a abordagem de Demerjian et al. (2012), que leva em consideração as características próprias da firma e as características de gestão do CFO. Em geral, empresas eficientes são aquelas que geram maiores receitas para um determinado conjunto de insumos. O resíduo dessa regressão é usada como proxy de capacidade gerencial do diretor financeiro e empregada para identificar o seu impacto na qualidade de resultado financeiro, representado aqui pelo volume de reapresentações dos relatórios contábeis, tomando como base os estudos de Dechow et al. (2010).

A contribuição deste trabalho para a literatura referente a qualidade de resultados e de eficiência e desempenho das firmas se dá ao estabelecer uma relação positiva entre a capacidade gerencial e a qualidade dos resultados. Segundo 
Demerjian et al. (2013), esta relação é importante para os comitês de governança corporativa ao considerar os custos e benefícios da contratação de CFOs mais hábeis, pois a capacidade gerencial além de afetar as operações afeta também a qualidade dos resultados reportados e, consequentemente, os atributos de preço de ações. Ainda, esse estudo serve de auxílio para entes reguladores, como a comissão de valores mobiliários (CVM), na determinação de irregularidades contábeis nas elaborações das demonstrações financeiras das companhias listadas, na determinação de refazimento e republicação das demonstrações financeiras e, consequentemente, na aplicação de multas e penalidades aos gestores.

Nas próximas seções serão desenvolvidas a revisão da literatura, o desenvolvimento da hipótese de pesquisa e a metodologia utilizada no estudo, bem como o relato dos resultados encontrados na pesquisa.

\section{REVISÃO DA LITERATURA E DESENHO DA HIPÓTESE}

Qualidade dos resultados é uma característica desejável dos relatórios contábeis, produzindo efeitos nas decisões de alocação de recursos de maneira eficiente. Sendo assim, o lucro é a principal variável de análise nos modelos de avaliação dos investimentos. Empresas com baixa qualidade dos resultados apresentam risco de informação, podendo assim, dar bases não fidedignas a elaboração dos relatórios contábeis e ocasionando consequências econômicas (Zhang, 2014). No caso específico sobre as consequências econômicas, tem-se o efeito da baixa qualidade de resultados no custo de capital. Por exemplo, Feldmann, Read \& Abdolmohammadi (2009) mostraram que as taxas de auditorias são mais altas para as empresas com maior número de reapresentações dos relatórios financeiros. Taxas mais altas de auditoria são uma resposta à crise de legitimidade, consequentemente há aumento do custo de capital. Com relação a correspondência entre a qualidade dos resultados e o custo de capital, Dechow et al. (2010), na sua revisão de literatura sobre as proxies de qualidade de resultados, documentaram uma associação inversa entre qualidade dos resultados e custo de capital. Como o propósito dos relatórios contábeis é o fornecimento de informações úteis para os tomadores de decisão, define-se resultado de alta qualidade aquele que representa de maneira completa, neutra e livre de erro o desempenho operacional da empresa (CPC 00).

Com relação ao efeito dos gerentes financeiros na empresa, Fee, Hadlock e Pierce (2013) identificaram que as saídas destes levam a grandes mudanças de políticas de gestão. Esta evidência sugere a existência de estilos individuais de gestão e que estes estilos gerenciais não são transferidos.

Nessa linha, Francis, Hasan, Park e Wu (2015) examinaram o efeito do gerente em certas políticas de relatórios financeiros e, como Fee et al. (2013), documentaram que as políticas de contabilização e divulgação das empresas variam de acordo com o CFO. Geiger e North (2006), mostraram que a contratação do CFO externo diminui o montante de accruals discricionários mais que a troca do CEO. Por outro lado, Li, Sun e Ettredge (2010) mostraram que a melhoria de opinião da SOX 404 só se dá na contratação de um CFO melhor qualificado. Esse comportamento permite aos pesquisadores documentarem um efeito específico do gerente na produção dos relatórios contábeis. Entretanto, esta abordagem é limitada aos gerentes que trocam de companhia entre as empresas da amostra. De maneira específica, as pesquisas de Aier, Comprix, Gunlock e Lee (2005) e Zhang (2014) examinaram se a qualidade dos resultados varia de acordo com as características gerenciais.

Por outro lado, Aier et al. (2005) documentaram uma associação entre os conhecimentos do CFO e a reapresentação dos relatórios financeiros, concluindo que existe uma relação inversa entre CFOs mais experientes e o volume de reapresentações, o que pode ser explicado pela relação entre a rotatividade do CFO, qualificação e sua compensação de bônus com as reformulações dos relatórios financeiros (Feldmann et al., 2009). Por outro lado, Zhang (2014) mostrou que empresas com recentes reapresentações dos relatórios financeiros são menos propensas a se tornarem alvo de aquisição. Adicionalmente, mostraram que para empresas com reapresentações contábeis que possuem ofertas públicas de aquisição, existe uma maior probabilidade de as ofertas de aquisição serem retiradas ou, ainda, o tempo de aquisição ser mais longo em comparação às empresas sem reapresentações. Assim, mantendo fixas as características de desempenho fundamentais das empresas formula-se a seguinte hipótese a ser testada: Habilidade da gestão financeira está positivamente associada a qualidade dos ganhos.

A hipóste desse trabalho se baseia na ideia de que os CFOs exercem um papel importante na qualidade dos resultados reportados, pois a elaboração dos relatórios financeiros são de sua responsabilidade. Jiang, Petroni e Wang (2010) demonstram que os CFOs desempenham um papel mais forte do que o CEOs no gerenciamento de resultados. A medida de qualidade considerada nesta pesquisa foi baseada no estudo de Dechow et al. (2010) e refere-se a existência de reapresentação dos relatórios contábeis como indicador de ganhos inconsistentes (Dichev, Graham, Harvey \& Rajgopal, 2013). O primeiro argumento para a escolha desta proxy de qualidade de resultados é que menor nível de reapresentação de relatórios financeiros indica ganhos mais persistentes, verificando a característica de neutralidade na apresentação fidedigna dos relatórios contábeis (Dechow et al., 2010; Richardson et al., 2005).

O segundo argumento é o vínculo entre os níveis de reapresentações dos relatórios financeiros e o valor absoluto de accruals totais extremos. Segundo Neelson e Skinner (2013), empresas com accruals totais extremos tendem a possuir accruals discricionários extremos e maior volume de reapresentação dos relatórios financeiros. Adicionalmente, Dichev et al. (2013) mostraram que 50\% dos accruals totais são oriundos de fatores não discricionários, como indústria e condições macroeconômicas. O percentual restante, produto do processo de geração dos accruals totais, é produto de escolhas discricionárias. Neste trabalho não estamos interessados no processo de escolhas contábeis com o fim de benefício próprio 
na elaboração dos relatórios financeiros e nos limitamos a reapresentação dos relatórios financeiros como proxy de qualidade da informação contábil baseando-se na hipótese das relações dadas nos trabalhos neste parágrafo supracitados.

O terceiro argumento é que nossa medida (reapresentação dos relatórios financeiros) é uma proxy de qualidade dos resultados na dimensão da resposta do investidor aos ganhos, seguindo a linha de pesquisas anteriores (Teoh \& Wong, 1999; Armstrong, Guay \& Weber, 2010). Para cumprir o objetivo desta pesquisa, a proxy de eficiência da firma aqui utilizada não é afetada pelas escolhas contábeis. Assim, a relação esperada entre reapresentação dos relatórios financeiros e a habilidade do gestor será negativa, caso contrário, os resultados podem não indicar esta relação devido a problemas, tais como amostra de reformulação de relatórios financeiros incluindo empresas que estão corrigindo erros não intencionais ou aplicando novos pronunciamentos retrospectivamente (CPC 23).

Assim, espera-se que o efeito da capacidade gerencial do CFO reflete menores níveis de reapresentações dos relatórios contábeis e, consequentemente, melhores níveis de qualidade da informação contábil. Os resultados da pesquisa ampliam a compreensão do efeito dos gestores na credibilidade dos relatórios contábeis, pois reformulações de números contábeis errôneos, principalmente de lucros, podem levar a perdas significativas como, por exemplo, o aumento do custo de capital. Consequentemente, uma melhor qualidade dos relatórios restaura a credibilidade dos investidores.

A contribuição teórica do estudo para a literatura dá-se ao oferecer uma melhor medida de habilidade do gestor CFO. Ao contrário de Demerjian et al. (2013), nossa proxy de eficiência é representada por uma melhor medida de desempenho na criação de valor. Veja que o uso de variáveis como lucro na criação de proxies de desempenho só representa uma parte da riqueza criada. Dechow et al. (2010) apontam que cuidados devem ser tomados na criação de uma proxy para medir eficiência da firma, pois esta não deve ser afetada pelo sistema contábil. Assim, oferecemos uma medida de eficiência mais robusta que as definições das proxies de eficiência utilizadas em estudos anteriores, como exemplo, ROA, ROE e a medida criada por Demerjian et al. (2013).

Portanto, para estimar a eficiência total da empresa, utiliza-se o valor adicionado total como outputs e como inputs o valor da distribuição do valor adicionado em pessoal e encargos, impostos, taxas, contribuições juros e aluguéis e, por último, juros sobre o capital próprio e dividendos, baseado na característica das informações da demonstração do valor adicionado (CPC 09). Dessa forma, a proxy de eficiência deste estudo representa uma melhor medida do quanto que a entidade agrega de valor aos insumos adquiridos.

\section{METODOLOGIA}

A amostra deste estudo é composta por todas as companhias abertas listadas na B3 Brasil, Bolsa e Balcão. As informações referentes a reapresentação dos relatórios financeiros foram extraídas das notas explicativas dos relatórios financeiros anuais. A informação de se as empresas foram auditadas por uma empresa de auditoria considerada como uma Big Four foi coletada manualmente dos dados econômicos financeiros na parte de informações relevantes do site da B3. O período analisado compreende os anos de 2010 a 2017. O período de estudo se justifica por compreender somente anos posteriores a adoção das normas internacionais de contabilidade no país. Os dados foram coletados anualmente e organizados em painel. Como explicado anteriormente, nossa medida de eficiência da empresa difere da proxy de Demerjian et al. (2013) em vários aspectos metodológicos, além das diferenças na escolha das variáveis para a criação da medida de desempenho da empresa.

Utiliza-se o método de Stochastic Production Frontier (SFA) para a estimação da eficiência das empresas, pois a análise envoltória de dados (DEA), usada por Demerjian et al. (2013), não lida com os erros de medição, como apontado por Aigner, Lovell e Schmidt (1997) baseado na ideia que os desvios da fronteira eficiente podem não estar inteiramente sobre o controle da empresa. Logo, faz sentido utilizar um método de estimação de eficiência que lide efetivamente com o ruído estatístico nos dados, motivado pela ideia que a medida de eficiência gerada pela estimativa é atribuível a empresa e a capacidade do CFO (Demerjian et al. 2013). Assim, o modelo teórico envolve uma função na qual o termo de erro contém dois componentes: um para explicar a ineficiência técnica e um para explicar os efeitos aleatórios. Este estudo, dado um conjunto de dados em painel, considera um modelo SFA especificado para painel como segue:

$$
\mathrm{Y}_{\mathrm{it}}=\alpha_{\mathrm{i}}+\beta^{\prime} \mathrm{X}_{\mathrm{it}}+\mathrm{v}_{\mathrm{it}}-\mathrm{u}_{\mathrm{it}} \text { onde } \mathrm{i}=1,2, \ldots . \mathrm{N} ; \mathrm{t}=1,2, \ldots . \mathrm{T}(1)
$$

onde $\mathrm{Y}_{\mathrm{it}}$ representa o logarítmo do valor adicionado total da i-ésima Decision Making Unit (DMU) no t-ésimo ano; $\mathrm{X}_{\mathrm{it}}$ é um vetor de valores da distribuição do valor adicionado em pessoal e encargos, impostos, taxas e contribuições, juros e aluguéis e, por último juros sobre o capital próprio e dividendos da i-ésima DMU no t-ésimo ano; $\beta$ é um vetor de parâmetros desconhecidos; $\alpha_{\mathrm{i}}$ são os efeitos específicos individuais das DMUs, $\mathrm{v}_{\mathrm{it}}$ é o termo de erro aleatório que é assumido como i.i.d. (independente e identicamente distribuído) com distribuição $\left(0, \sigma_{\mathrm{v}}\right)$, e independente de $\mathrm{u}_{\mathrm{it}}$ que é a ineficiência técnica. Como o interesse está na parcela atribuível a capacidade gerencial, toma-se como capacidade do CFO o termo do erro aleatório, chamado neste estudo de Mhabil. Vale ressaltar que o modelo apresentado anteriormente é um fixed effects production frontier model proposto por Greene (2004), estimado mediante máximo verossimilhança e especificado de tal forma que todos os efeitos invariantes no tempo são capturados por $\alpha_{\mathrm{i}}$ (componente específico individual de cada DMU) e a ineficiência técnica varia livremente ao longo do tempo. Veja que o objetivo primário não é a estimativa dos parâmetros do modelo, mas a estimativa do termo de ineficiência e, portanto, estimativa da eficiência técnica. Logo, de acordo com a abordagem de Demerjian et al. (2013), este resíduo representa a capacidade do CFO, denominada neste estudo como Mhabil. 
Em seguida, para determinar a relação da habilidade do CFO na qualidade dos resultados será estimada a seguinte equação:

$$
\operatorname{Reap}_{1 \mathrm{t}}=\alpha_{1}+\alpha_{1} \text { Mhabil }_{1 \mathrm{t}}+\alpha_{2} \text { Size }_{1 \mathrm{t}}+\alpha_{3} \text { Vendas }_{1 \mathrm{t}}+\alpha_{4} \mathrm{FCO}_{\mathrm{it}}+\alpha_{5} \text { BigFour }_{1 \mathrm{tt}}+\alpha_{6} \Delta \mathrm{LUC}_{\mathrm{tt}}+\varepsilon_{1 \mathrm{t}}
$$

onde Mhabil é a eficiência técnica estimada da equação 1, Size é o logaritmo natural do ativo total, Vendas é a variação das contas a receber do ano t em relação ao ano anterior dividido pelo ativo total, FCO é o fluxo de caixa operacional dividido pelo ativo total, BigFour é uma dummy que toma o valor de 1 se foi auditada por uma empresa de auditoria considerada como Big Four e 0 caso contrário, $\triangle$ LUC é a variação do lucro do ano t em relação ao ano anterior dividido pelo ativo total, Reap é uma dummy que toma o valor de 1 se a empresa teve reapresentação dos relatórios contábeis e 0 caso contrário.

A Equação 2 foi estimada mediante o modelo de regressão probit em painel, uma vez que a variável dependente do estudo é uma variável dummy que assume valor 1 ou 0 . De maneira específica, foi utilizado o generalized estimating equation (GEE) approach com variância robusta, porque o trabalho está focado em descobrir o efeito médio da variável Mhabil em relação ao efeito específico individual. Justifica-se a escolha dessa abordagem devido a probabilidade de uma empresa de reapresentar seus relatórios financeiros estar correlacionadas a algumas características das mesmas (por exemplo, tamanho), violando as hipóteses de independência feitas pelos procedimentos tradicionais de regressão.

Mediante o modelo apresentado, espera-se que os gestores com maior capacidade gerencial obtenham menores volumes de reapresentações dos resultados financeiros da empresa e, portanto, melhor nível de qualidade de resultados. Isto acontece pois a qualidade dos resultados é uma função do desempenho da firma e, por sua vez, da parte do desempenho correlacionada com a equipe da gerência responsável.

\section{ANÁLISE DOS DADOS}

Após o tratamento da base de dados, o total de observações foi de 1839 compreendendo o período 2010 até 2017. Do total de observações, 360 (19,57\%) são referentes a reapresentação dos relatórios financeiros e 1479 (80,43\%) de não reapresentação. Os dados foram winsorizados ao nível de $1 \%$.

Na Tabela 1 são apresentadas as estatísticas descritivas.

Tabela 1 - Estatística descritiva

\begin{tabular}{c|c|c|c|c|c}
\hline Variável & Observações & Média & Desvio Padrão & Mínimo & Máximo \\
\hline Reap & 1839 & 0,1958 & 0,3969 & 0 & 1 \\
Mhabil & 1839 & $-2,5000$ & 0,4104 & $-4,4147$ & $-1,5928$ \\
Size & 1839 & 14,5558 & 2,0292 & 6,6503 & 18,8053 \\
BigFour & 1839 & 0,8989 & 0,3016 & 0 & 1 \\
FCO & 1839 & 0,0006 & 0,0611 & $-0,2513$ & 0,2349 \\
LLUC & 1839 & $-0,0053$ & 0,2777 & $-1,4923$ & 1,2374 \\
Vendas & 1839 & $-0,0012$ & 0,0743 & $-0,3547$ & 0,2944 \\
\hline
\end{tabular}

Nota: Mhabil é a eficiência técnica estimada da Equação 1, Size é o logaritmo natural do ativo total, Vendas é a variação das contas a receber do ano t em relação ao ano anterior dividido pelo ativo total, FCO é o fluxo de caixa operacional dividido pelo ativo total, BigFour é uma dummy que toma o valor de 1 se foi auditada por uma empresa de auditoria considerada como Big Four e 0 caso contrário, $\triangle$ LUC é a variação do lucro do ano t em relação ao ano anterior dividido pelo ativo total, Reap é uma dummy que toma o valor de 1 se a empresa teve reapresentação dos relatórios contábeis e 0 caso contrário.

Fonte: Base de dados.

Os resultados indicam uma grande heterogeneidade dos dados que compõem a amostra de estudo. A variável size, que representa o tamanho das firmas, apresentou um considerável desvio padrão indicando que existe dispersão no que diz respeito ao tamanho das firmas aqui estudadas. Quando analisadas as variáveis relacionadas a lucratividade das empresas e ao volume das empresas, pode-se verificar que as empresas estudadas tiveram resultados médios negativos dentro do período da amostra. A variável que representa o fluxo de caixa operacional das empresas apresentou a maior variação em torno da média, sugerindo existir grande diversidade no que se refere a movimentação das operações das empresas.

Para uma melhor análise da eficiência oriunda da eficiência do CFO e sua equipe, fez-se a segregação da média e do desvio padrão das variáveis que representam a qualidade da informação gerada (Reap) e a habilidade do CFO (Mhabil). O objetivo é analisar se a dispersão desses dados é dada entre cada empresa da amostra ou oriunda da mesma empresa no transcorrer do tempo. 
Os resultados da segregação da média das variáveis Reap e Mhabil são apresentados na Tabela 2.

Tabela 2 - Estatística descritiva: Decomposição das variáveis Reap e Mhabil

\begin{tabular}{|c|c|c|c|c|c|}
\hline Variável & & Média & Desvio Padrão & Mínimo & Máximo \\
\hline \multirow{3}{*}{ Reap } & Overall & 0,1958 & 0,3969 & 0 & 1 \\
\hline & Between & & 0,1656 & 0,0000 & 0,8571 \\
\hline & Within & & 0,3620 & $-0,6614$ & 1,0529 \\
\hline \multirow{3}{*}{ Mhabil } & Overall & $-2,5000$ & 0,4104 & $-4,4147$ & $-1,5928$ \\
\hline & Between & & 0,2675 & $-3,8419$ & $-1,7515$ \\
\hline & Within & & 0,3123 & $-4,1929$ & $-1,5823$ \\
\hline
\end{tabular}

Nota: Mhabil é o resíduo da Equação 1 e Reap é uma dummy que toma o valor de 1 se a empresa reapresentou as demonstrações contábeis no ano em questão e 0 caso contrário.

Fonte: Base de dados.

Pode-se verificar, de acordo com os resultados apresentados, que o desvio padrão é muito maior de cada empresa com relação ao tempo do que as entre empresas propriamente ditas, resultando em que a capacidade do CFO é tenue quando observada uma empresa em comparação a outra. A variável dependente (Reap) possue um comportamento similar a variável independente que busca medir a habilidade do gestor CFO (Mhabil).

Com o intuito de verificar se existe diferenças na eficiência técnica do gestor CFO (Mhabil) entre as empresas que reapresentaram os seus relatórios contábeis para aquelas que não representaram suas demonstrações, foi rodado um teste de médias para duas populações. Entretanto, como a variância populacional é desconhecida, foi feito um teste de diferença de variâncias populacionais, para averiguar se estas são iguais ou diferentes. Os resultados são apresentados na Tabela 3 e indicam que as variâncias populacionais entre os dois grupos são diferentes.

Tabela 3 - Teste de diferença de variâncias

\begin{tabular}{c|c|c|c}
\hline & Empresas com reapresentação & Empresas sem reapresentação & \\
\hline Variável & Desvio Padrão & Desvio Padrão & P-valor \\
Mhabil & 0,5239 & 0,3763 & 0,0000 *** \\
\hline
\end{tabular}

Nota: ${ }^{\star \star \star}$ significativo a $1 \%$.

Fonte: Base de dados.

Em seguida, levando em consideração os resultados da Tabela 3, foi rodado um teste de diferença de médias, com o intuito de verificar se existem diferenças estatisticamente significantes entre as médias dos desempenhos de CFOs das empresas que reapresentaram seus relatórios contábeis e as que não. Os resultados do teste de diferenças de médias são apresentados na Tabela 4.

Tabela 4 - Teste de diferenças de médias

\begin{tabular}{c|c|c|c|c} 
& Empresas com reapresentação & Empresas sem reapresentação & & \\
\hline Variável & Média & Média & Diferenças & P-valor \\
Mhabil & $-2,5617$ & $-2,4850$ & 0,0766 & $0,0092^{\star \star \star}$ \\
\hline
\end{tabular}

Nota: ${ }^{\star \star \star}$ significativo a $1 \%$.

Fonte: Base de dados.

Como visto nos resultados da Tabela 4, as empresas que não possuem reapresentação de relatórios contábeis tem Mhabil médio estatisticamente maior do que as empresas que reapresentam seus relatórios contábeis. Estes resultados já dão um sinal de que a capacidade da gerência afeta de maneira diferenciada a probabilidade de reapresentação dos relatórios contábeis.

Na Tabela 5 são apresentados os resultados das correlações entre as variáveis de interesse. 
Tabela 5 - Matriz de correlação

\begin{tabular}{c|c|c|c|c|c|c|c}
\hline & Reap & Mhabil & Size & BigFour & FCO & $\Delta$ LUC & Vendas \\
\hline Reap & 1,0000 & & & & & \\
Mhabil & $-0,0741^{\star \star \star}$ & 1,0000 & & & & \\
Size & 0,0371 & $0,3292^{\star \star \star}$ & 1,0000 & & & \\
BigFour & 0,0200 & $0,1888^{\star \star \star}$ & $0,1829 \star \star \star$ & 1,0000 & & \\
FCO & 0,0216 & 0,0141 & $0,0522^{\star \star}$ & $-0,0066$ & 1,0000 & & \\
LLUC & $-0,0003$ & 0,013 & 0,0224 & $-0,0185$ & $0,0888^{\star \star *}$ & 1,0000 & \\
Vendas & $0,0605^{\star \star \star}$ & 0,0043 & 0,0065 & $-0,014$ & $-0,0001$ & $0,0924^{\star \star \star}$ & 1,0000 \\
\hline
\end{tabular}

Nota: Mhabil é a eficiência técnica estimada da Equação 1, Size é o logaritmo natural do ativo total, Vendas é a variação das contas a receber do ano t em relação ao ano anterior dividido pelo ativo total, FCO é o fluxo de caixa operacional dividido pelo ativo total, BigFour é uma dummy que toma o valor de 1 se foi auditada por uma empresa de auditoria considerada como Big Four e 0 caso contrário, $\triangle \mathrm{LUC}$ é a variação do lucro do ano t em relação ao ano anterior dividido pelo ativo total, Reap é uma dummy que toma o valor de 1 se a empresa teve reapresentação dos relatórios contábeis e 0 caso contrário.

Nota: $* \star \star, * \star * *$ significativo a $1 \%, 5 \%$ e $10 \%$, respectivamente.

Fonte: Base de dados.

Como o esperado, existe uma correlação positiva entre a parcela da eficiência atribuível a equipe de CFOs (Mhabil) e tamanho da firma (size). Adicionalmente, também existe uma correlação positiva entre BigFour e Mhabil. Portanto, empresas grandes e auditadas por uma empresa BigFour está relacionada positivamente à capacidade do CFO. Os resultados demonstram que existe uma correlação positiva e tênue entre as variáveis ativo total, lucro e fluxo de caixa operacional, isto é, empresas de grande porte tendem a apresentar maiores fluxos de caixa e maiores lucros. Por outro lado, com relação a nossa variável proxy para qualidade informacional (Reap) existe uma correlação positiva com volume de vendas (Vendas) e uma correlação negativa com a habilidade do gestor CFO (Mhabil). Assim, a medida de qualidade de resultados de Dechow et. al. (2010), Reap está negativamente associada com a habilidade do CFO, consistente com Demerjian et. al (2013) e os resultados da tabela 5.

Por último foi estimado o modelo da equação 2 e os resultados se encontram na Tabela 6.

Tabela 6 - Resultado da estimação da Equação 2

\begin{tabular}{|c|c|c|c|c|}
\hline Variáveis & Coefiente & $\begin{array}{c}\text { Desvio padrão } \\
\text { Robusto }\end{array}$ & $\mathbf{z}$ & $P>|z|$ \\
\hline Mhabil & -0.3884 & 0.1055 & -3.6800 & $0.0000 * * *$ \\
\hline Size & 0.0482 & 0.0232 & 2.0800 & $0.0380 * \star$ \\
\hline Big4 & 0.1396 & 0.1439 & 0.9700 & 0.3320 \\
\hline FCO & 0.4474 & 0.6147 & 0.7300 & 0.4670 \\
\hline$\Delta$ Luc & -0.0304 & 0.1505 & -0.2000 & 0.8400 \\
\hline Vendas & 1.3495 & 0.4228 & 3.1900 & $0.0010 * \star *$ \\
\hline Constante & -2.6677 & 0.4736 & -5.6300 & $0.0000^{* * *}$ \\
\hline $\begin{array}{l}\text { Número de } \\
\text { observações }\end{array}$ & 1839 & & & \\
\hline Wald $X^{2}$ & 26,58 & & & \\
\hline Prob $>X^{2}$ & 0,0002 & & & \\
\hline
\end{tabular}

Mhabil é a eficiência técnica estimada da Equação 1, Size é o logaritmo natural do ativo total, Vendas é a variação das contas a receber do ano t em relação ao ano anterior dividido pelo ativo total, FCO é o fluxo de caixa operacional dividido pelo ativo total, BigFour é uma dummy que toma o valor de 1 se foi auditada por uma empresa de auditoria considerada como Big Four e 0 caso contrário,

$\triangle$ LUC é a variação do lucro do ano t em relação ao ano anterior dividido pelo ativo total, Reap é uma dummy que toma o valor de 1 se a empresa teve reapresentação dos relatórios contábeis e 0 caso contrário.

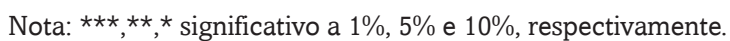

Fonte: Base de dados 
O resultado do teste Wald indica que ao menos um dos coeficientes do modelo se mostra estatisticamente diferente de zero. Estes resultados demonstram que o modelo prediz a chance de uma empresa reapresentar seus relatórios financeiros de maneira satisfatória.

Com relação a nossa variável de interesse Mhabil, se uma empresa aumentar a capacidade da equipe CFOs, a probabilidade esperada de reapresentação dos relatórios financeiros diminui, mantendo todas as outras variáveis do modelo como controle no modelo. Ou seja, quanto mais eficiente o CFO, menos provável que a empresa reapresente seus relatórios financeiros $\left(\alpha_{1}=-0.38 ; p<0.01\right)$. Dessa forma, quanto maior a capacidade do CFO e sua equipe, menor será a probabilidade de reapresentação dos relatórios financeiros previsto e, consequentemente, maior a qualidade dos resultados para estas empresas.

Cabe salientar neste ponto, que o a variação do lucro, o fato de uma empresa ser auditada por uma empresa de auditoria BigFour e o fluxo de caixa operacional não apresentaram ser fatores importantes para o cálculo da probabilidade de uma empresa reapresentar ou não seus resultados financeiros. Isso pode ser explicado devido às reapresentações analisadas neste período não estarem relacionadas ao refazimento das informações financeiras por apresentações erradas no cálculo dos lucros apresentados por estas empresas no caso da variação do lucro ( $\Delta$ Luc). Ainda, pode indicar que empresas de auditoria podem estar mais preocupadas com a materialidade, justificando o efeito nulo da variável na probabilidade de reapresentação.

Os outros 2 determinantes de reapresentação dos relatórios financeiros foram a variável relacionada aos ativos da empresa e venda, demonstrando que empresas de maior porte e maior volume de vendas possuem uma maior probabilidade a reapresentar seus relatórios financeiras quando comparadas as empresas de menor tamanho e menor variação de vendas. Assim, os resultados, corroboram os sinais e as relações $\left(\alpha_{1}=-, \alpha_{2}=+, \alpha_{3}=+; p<0.01, p<0.05, p<0.01\right)$ dos determinantes do volume de reapresentação e os resultados aqui encontrados vão de acordo com os resultados de Demerjian et al (2013).

\section{CONSIDERAÇÕES FINAIS}

Este estudo teve por propósito estudar o efeito da capacidade gerencial na eficiência das firmas listadas na B3, analisando a relevância da capacidade gerencial do CFO e sua equipe na qualidade dos resultados contábeis. Ao ser feita a revisão da literatura, constatou-se que a maior parte dos trabalhos focados nas análises da qualidade da informação contábil e capacidade do gestor se concentram nas relações CEOs e qualidade da informação contábil.

Os resultados encontrados mostram uma relação negativa e significativa entre Mhabil e o nível de reapresentação dos relatórios financeiros, demonstrando que CFOs mais capazes implicam em menores chances de reapresentação dos resultados financeiros. As evidências empíricas demonstram que a escolha do gestor CFO é importante para o mercado de capitais, pois reformulações refletem erros que levam os investidores a revisar suas crenças sobre a precisão das informações associadas aos ganhos da empresa (Kravet \& Shevlin, 2010).

Os resultados estão alinhados com Demerjian, Lev e Mcvay (2012). Demerjian et al. (2013) encontraram relação negativa entre a capacidade da equipe do CFO e a variável associada a reapresentação dos relatórios financeiros e relação positiva e significativa quando analisados os efeitos do fluxo de caixa operacional e ativo total, mostrando a importância para os comitês de governança corporativa ao considerar os custos e benefícios da contratação de CFOs mais capacitados. Pesquisas demonstram que o retorno médio de uma ação cai 9,2\% quando ocorre o anúncio de reapresentação dos relatórios financeiros e $20 \%$ quando o motivo está vinculado a litígios (Palmrose et al. 2004).

A contribuição deste trabalho para a literatura referente a qualidade de resultados e de eficiência e desempenho das firmas se dá ao estabelecer uma relação negativa entre a probabilidade de reapresentação dos relatórios financeiros e a capacidade da equipe do CFO e, portanto, uma relação positiva entre a capacidade da equipe do CFO e a qualidade dos resultados contábeis. Os resultados da pesquisa estão de acordo com os resultados anteriormente demonstrados na literatura sobre relevância da capacidade da equipe do CFO.

Os resultados desta pesquisa devem ser interpretados levando em consideração a limitação da variável reapresentação dos relatórios financeiros como uma proxy do intentional misstatement, pelo fato de não saber qual a proporção intencional e não intencional, pois as reapresentações podem ser solicitadas pela CVM, pela própria firma ou pela empresa de auditoria de maneira que isto pode trazer um pouco de ruído na análise das relações. Hennes, Leones e Miller (2008) argumentam que distinguir os erros das irregularidades aumenta o poder dos testes relacionados a reapresentação das informações contábeis. Segundo Leone e Liu (2010), CFOs são mais propensos a assumir a culpa quando o CEO é um dos fundadores, assim uma outra observação e que está fora do copo de estudo desta pesquisa é a probabilidade de que CFOs cometam intentional misstatement em resposta à pressão recebida dos CEOs e não em benefício pessoal.

Em pesquisa futura, sugere-se que seja avaliada o efeito da capacidade CFO e outras proxies de qualidade da informação contábil no intuito de analisar se os resultados convergem, apresentando, assim insight sobre a importância de contratar CFOs mais capacitados, resultando em melhor custo benefício para a empresa.

\section{REFERÊNCIAS}

Aier, J. K., Comprix, J., Gunlock, M. T., \& Lee, D. (2005). The financial expertise of CFOs and accounting restatements. Accounting Horizons, 19(3), 123-135. https://doi.org/10.2308/acch.2005.19.3.123 
Aigner, D., Lovell, C. K., \& Schmidt, P. (1977). Formulation and estimation of stochastic frontier production function models. Journal of econometrics, 6(1), 21-37. https://doi.org/10.1016/0304-4076(77)90052-5

Amel-Zadeh, A., \& Zhang, Y. (2014). The economic consequences of financial restatements: Evidence from the market for corporate control. The Accounting Review, 90(1), 1-29. https://doi.org/10.2308/accr-50869

Armstrong, C. S., Guay, W. R., \& Weber, J. P. (2010). The role of information and financial reporting in corporate governance and debt contracting. Journal of Accounting and Economics, 50(2-3), 179-234. https://doi.org/10.1016/j.jacceco.2010.10.001

Becker, C. L., DeFond, M. L., Jiambalvo, J., \& Subramanyam, K. R. (1998). The effect of audit quality on earnings management. Contemporary accounting research, 15(1), 1-24. https://doi.org/10.1111/j.1911-3846.1998.tb00547.x

Bertrand, M., \& Schoar, A. (2003). Managing with style: The effect of managers on firm policies. The Quarterly journal of economics, 118(4), 1169-1208. https://doi.org/10.1162/003355303322552775

Dechow, P. M., \& Dichev, I. D. (2002). The quality of accruals and earnings: The role of accrual estimation errors. The accounting review, 77(s-1), 35-59. https://doi.org/10.2308/accr.2002.77.s-1.35

Dechow, P., Ge, W., \& Schrand, C. (2010). Understanding earnings quality: A review of the proxies, their determinants and their consequences. Journal of accounting and economics, 50(2-3), 344-401. https://doi.org/10.1016/j.jacceco.2010.09.001

Demerjian, P., Lev, B., \& McVay, S. (2012). Quantifying managerial ability: A new measure and validity tests. Management science, 58(7), 1229-1248. http://dx.doi.org/10.1287/mnsc.1110.1487

Demerjian, P. R., Lev, B., Lewis, M. F., \& McVay, S. E. (2013). Managerial ability and earnings quality. The Accounting Review, 88(2), 463-498. : http://dx.doi.org/10.2308/accr-50318

Dichev, I. D., Graham, J. R., Harvey, C. R., \& Rajgopal, S. (2013). Earnings quality: Evidence from the field. Journal of Accounting and Economics, 56(2-3), 1-33. http://dx.doi.org/10.1016/j.jacceco.2013.05.004

Doyle, J. T., Ge, W., \& McVay, S. (2007). Accruals quality and internal control over financial reporting. The accounting review, 82(5), 1141-1170. https://doi.org/10.2308/accr.2007.82.5.1141

Feldmann, D. A., Read, W. J., \& Abdolmohammadi, M. J. (2009). Financial restatements, audit fees, and the moderating effect of CFO turnover. Auditing: A Journal of Practice \& Theory, 28(1), 205-223. https://doi.org/10.2308/aud.2009.28.1.205

Feroz, E. H., Park, K., \& Pastena, V. S. (1991). The financial and market effects of the SEC's accounting and auditing enforcement releases. Journal of accounting research, 29, 107-142. http://hdl.handle.net/10.2307/2491006

Fee, C. E., Hadlock, C. J., \& Pierce, J. R. (2013). Managers with and without style: Evidence using exogenous variation. The Review of Financial Studies, 26(3), 567-601. https://doi.org/10.1093/rfs/hhs131

Francis, B., Hasan, I., Park, J. C., \& Wu, Q. (2015). Gender differences in financial reporting decision making: Evidence from accounting conservatism. Contemporary Accounting Research, 32(3), 1285-1318. https://doi.org/10.1111/1911-3846.12098

Gong, Y., Law, K. S., Chang, S., \& Xin, K. R. (2009). Human resources management and firm performance: The differential role of managerial affective and continuance commitment. Journal of Applied Psychology, 94(1), 263. https://doi.org/10.1037/a0013116

Greene, W. (2004). Distinguishing between heterogeneity and inefficiency: stochastic frontier analysis of the World Health Organization's panel data on national health care systems. Health economics, 13(10), 959-980. https://doi.org/10.1002/hec.938

Hribar, P., \& Craig Nichols, D. (2007). The use of unsigned earnings quality measures in tests of earnings management. Journal of Accounting Research, 45(5), 1017-1053.

Klein, A. (2002). Audit committee, board of director characteristics, and earnings management. Journal of accounting and economics, 33(3), 375-400.

Maestri, C., \& Malaquias, R. (2018). Aspectos do gestor, alocação de carteiras e desempenho de fundos no Brasil. Revista Contabilidade \& Finanças, 29(76), 82-96. https://doi.org/10.1590/1808-057x201804590

Matos, P. R. F., Penna, C. M., \& Silva, A. B. G. (2015). Fundos Mútuos de Investimento em Ações no Brasil: Incentivos, Gestão e Convergência. Brazilian Business Review, 12(2), 115-149. http://dx.doi.org/10.15728/bbr.2015.12.2.6

McNichols, M., \& Wilson, G. P. (1988). Evidence of earnings management from the provision for bad debts. Journal of accounting research, 1-31. https://doi.org/10.2307/2491176

Nelson, M. W., \& Skinner, D. J. (2013). How should we think about earnings quality? A discussion of "Earnings quality: Evidence from the field". Journal of Accounting and Economics, 56(2-3), 34-41. http://dx.doi.org/10.1016/j.jacceco.2013.10.003

Palmrose, Z. V., \& Scholz, S. (2004). The circumstances and legal consequences of non-GAAP reporting: Evidence from restatements. Contemporary Accounting Research, 21(1), 139-180. https://doi.org/10.1506/WBF9-Y69X-L4DX-JMV1

Plumlee, M., \& Yohn, T. L. (2010). An analysis of the underlying causes attributed to restatements. Accounting Horizons, 24(1), 4164. https://doi.org/10.2308/acch.2010.24.1.41

Teoh, S. H., \& Wong, T. J. (1993). Perceived auditor quality and the earnings response coefficient. Accounting Review, $346-366$. https://doi.org/10.2308/accr-10612

$\mathrm{Xu}$, Y., \& Zhao, L. (2016). An investigation of financial expertise improvement among CFOs hired following restatements. American Journal of Business, 31(2), 50-65. https://doi.org/10.1108/AJB-07-2015-0022 